\title{
Modulus and Phase of the Reduced Logarithmic Derivative of the Hankel Function
}

\author{
By Andrés Cruz and Javier Sesma
}

\begin{abstract}
The modulus and phase of the reduced logarithmic derivative of the Hankel function$$
z H_{\nu}^{(1) \prime}(z) / H_{\nu}^{(1)}(z)
$$

for complex variable $z$ and real order $\nu$, are investigated. Special attention is paid to the location of saddle points and their trajectories as $\nu$ varies.
\end{abstract}

1. Introduction. The solution of the Schrödinger equation with optical potentials, like those used to describe the low-energy nucleus-nucleus or hadron-nucleus interaction, is usually obtained by matching the reduced logarithmic derivatives of the internal and external wave-functions at the edge of the potential. Inside, the wave-function depends on the details of the potential; outside, it is proportional to the Hankel function. A good knowledge of the reduced logarithmic derivative of the Hankel function

$$
F_{\nu}(z)=z H_{\nu}^{(1) \prime}(z) / H_{\nu}^{(1)}(z)
$$

is of great help both for the speedy approximate solution of the Schrödinger equation and for the analysis of singularities of the $S$ matrix.

The purpose of this paper is to discuss the main features of the modulus and phase of $F_{\nu}(z)$ for complex variable $z$ and real order $\nu$. From the recurrence relations for the Hankel functions [4, Eq. 9.1.27]

$$
\begin{aligned}
& H_{\nu}^{(1) \prime}(z)=-H_{\nu+1}^{(1)}(z)+(\nu / z) H_{\nu}^{(1)}(z), \\
& H_{\nu}^{(1) \prime}(z)=H_{\nu-1}^{(1)}(z)-(\nu / z) H_{\nu}^{(1)}(z),
\end{aligned}
$$

and the property [4, Eq. 9.1.6]

$$
H_{-\nu}^{(1)}(z)=\exp \{i \pi \nu\} H_{\nu}^{(1)}(z),
$$

it is easy to prove the symmetry of $F_{\nu}(z)$ with respect to the sign of its order,

$$
F_{-\nu}(z)=F_{\nu}(z) \text {. }
$$

We need, therefore, to consider only nonnegative values of $\nu$ in our discussion.

As is well known, the Hankel function is multivalued in its variable. By using its analytic continuation formula [4, Eq. 9.1.37] and the Wronskian relation for $H_{\nu}^{(1)}(z)$

Received January 18, 1983.

1980 Mathematics Subject Classification. Primary 33A40; Secondary 65H05, 81F10.

Key words and phrases. Hankel functions, modulus and phase of the reduced logarithmic derivative, quantum potential scattering. 
and $H_{\nu}^{(2)}(z)$ [4, Eq. 9.1.17], one obtains

$$
F_{\nu}\left(z e^{i \pi m}\right)=F_{\nu}(z)+4 i e^{-i \pi \nu} \sin (m \nu \pi) / \pi \sin (\nu \pi) H_{\nu}^{(1)}(z) H_{\nu}^{(1)}\left(z e^{i \pi m}\right),
$$

$m$ integer,

which shows that $F_{\nu}(z)$ is also a multivalued function of $z$, except in the case of half-integer $\nu$ (physical values of the angular momentum $l=\nu-1 / 2$ ).

Symmetries of $F_{\nu}(z)$ in the complex $z$ plane can be found by making use of the relations [4, Eqs. 9.1.39 and 9.1.40]

$$
\begin{aligned}
& H_{\nu}^{(1)}\left(z e^{i \pi}\right)=e^{-i \pi} e^{-i \pi \nu} H_{\nu}^{(2)}(z), \\
& H_{\nu}^{(2)}(z)=\overline{H_{\nu}^{(1)}(\bar{z})}, \quad \text { for real } \nu,
\end{aligned}
$$

from which it follows that

$$
H_{\nu}^{(1)}\left(z e^{i \pi}\right)=e^{-i \pi} e^{-i \pi \nu} \overline{H_{\nu}^{(1)}(\bar{z})}, \quad \nu \text { real. }
$$

This equation allows one to obtain

$$
F_{\nu}\left(z e^{i \pi}\right)=\overline{F_{\nu}(\bar{z})}, \quad \nu \text { real, }
$$

which relates the moduli and phases of $F_{\nu}(z)$ at points located symmetrically with respect to the vertical axis, but in different Riemann sheets. If the $z$ plane is cut along the negative real semiaxis, (1.9) shows that $F_{\nu}(z)$ takes complex conjugate values at symmetrical points of the first and second quadrants of the principal branch, $-\pi<\arg z \leqslant \pi$. Moreover, $F_{\nu}(z)$ is real on the positive imaginary semiaxis, $\arg z=\pi / 2$. For half-integer values of $\nu, F_{\nu}(z)$ is singlevalued and, due to (1.9), takes complex conjugate values at points symmetrical with respect to the imaginary axis. Of course, $F_{\nu}(z)$ is then real also on the negative imaginary semiaxis.

Another symmetry property of $F_{\nu}(z)$ can be obtained in the case of $3 \nu$ being integer, $\nu$ noninteger. From the analytic continuation formula [4, Eq. 9.1.37] for $H_{\nu}^{(1)}(z)$ and by using (1.7), one obtains

$$
F_{\nu}\left(z e^{-i 2 \pi}\right)=\frac{\sin (3 \pi \nu) z H_{\nu}^{(1) \prime}(z)+e^{-i \pi \nu} \sin (2 \pi \nu) \overline{\bar{z} H_{\nu}^{(1) \prime}(\bar{z})}}{\sin (3 \pi \nu) \dot{H}_{\nu}^{(1)}(z)+e^{-i \pi \nu} \sin (2 \pi \nu) \overline{H_{\nu}^{(1)}(\bar{z})}},
$$

that, in the case under consideration, reduces to

$$
F_{\nu}\left(z e^{-i 2 \pi}\right)=\overline{F_{\nu}(\bar{z})}, \quad 3 \nu \text { integer, } \nu \text { noninteger. }
$$

This relation indicates that $F_{\nu}(z)$ takes complex conjugate values at symmetrical (with respect to the real axis) points of the quadrants $-3 \pi / 2 \leqslant \arg z \leqslant-\pi$ and $-\pi \leqslant \arg z \leqslant-\pi / 2$. Consequently, $F_{\nu}(z)$ is real on the negative real semiaxis, $\arg z=-\pi$.

Although our discussion will be limited to the logarithmic derivative of the first Hankel function $H_{\nu}^{(1)}(z)$, it can be extended to that of the second one, $H_{\nu}^{(2)}(z)$, through the relation

$$
z H_{\nu}^{(2) \prime}(z) / H_{\nu}^{(2)}(z)=\overline{F_{\nu}(\bar{z})}, \quad \nu \text { real, }
$$

easily obtained from (1.7).

In Section 2 we study the location of zeros, poles and saddle points of $F_{\nu}(z)$. Plots of its modulus and phase for several values of $\nu$ are presented in Section 3. In view of 
the multivaluedness of $F_{\nu}(z)$, we cut the complex $z$ plane along the positive imaginary semiaxis and restrict our discussion to the Riemann sheet $-3 \pi / 2<\arg z$ $\leqslant \pi / 2$.

2. Zeros, Poles and Saddle Points. In the construction of a chart of the modulus and phase of $F_{\nu}(z)$ it is of great aid to know the location of its zeros and poles, where the constant-phase lines converge. Obviously, the zeros and poles of $F_{\nu}(z)$ correspond to the zeros of $H_{\nu}^{(1) \prime}(z)$ and $H_{\nu}^{(1)}(z)$, respectively. These have been extensively studied, for real $\nu$, in a recent paper [2].

Apart from zeros and poles, constant-phase (or modulus) lines do not intersect except at the saddle points, where two constant-phase lines (and two constant-modulus lines) meet. Saddle points are characterized by the relation

$$
d F_{\nu}(z) / d z=0
$$

that, in view of the differential equation satisfied by $F_{\nu}(z)$,

$$
z d F_{\nu}(z) / d z+\left[F_{\nu}(z)\right]^{2}+z^{2}-\nu^{2}=0
$$

is equivalent to

$$
\left[F_{\nu}(z)\right]^{2}=\nu^{2}-z^{2} \text {. }
$$

We have used this relation to analyze the location of saddle points in the complex $z$ plane as a function of the order $\nu$. Two infinite sets of saddle points are found, lying respectively above ("upper" saddle points) and below ("lower" saddle points) the zeros and poles of $F_{\nu}(z)$. In Figure 1 we show the trajectories followed by the saddle points as the order $\nu$ changes through real values. For $\nu=n+1 / 2\left(n\right.$ integer) $F_{\nu}(z)$ reduces to a quotient of polynomials and presents only $n$ saddle points of the "lower" type. With the exception of these, all saddle points go to infinity as $\nu$ tends to $n+1 / 2$. Their behavior can be deduced from (2.3) by using asymptotic expansions [4, Eqs. 9.2.7 and 9.2.8] for the Hankel functions and following a procedure similar to that used in [2] for the zeros of $H_{\nu}^{(1)}(z)$. The real and imaginary parts of the saddle points (labelled by the integer $s$ ) turn out to satisfy, for the " upper' ones,

$$
\begin{gathered}
x_{s} \simeq \pi / 4-s \pi-(1 / 2) \arg \xi \\
y_{s} \simeq(1 / 2) \log |\xi|, \\
\xi \equiv\left[1-4 \cos ^{2}(\nu \pi)\right]\left[4 z_{s} e^{i \pi 3 / 2}+2 \nu^{2}+1 / 2\right] / 2 \cos (\nu \pi),
\end{gathered}
$$

and for the "lower" ones

$$
\begin{gathered}
x_{s} \simeq \pi / 4-s \pi-(1 / 2) \arg \zeta \\
y_{s} \simeq(1 / 2) \log |\zeta| \\
\zeta \equiv\left[1-4 \cos ^{2}(\nu \pi)\right] /\left[4 z_{s} e^{i \pi / 2}+2 \nu^{2}+1 / 2\right] 2 \cos (\nu \pi)
\end{gathered}
$$

These equations do not give explicitly the positions of the saddle points because $z_{s} \equiv x_{s}+i y_{s}$ appears also on the right-hand sides. However, they allow a comparison of the location of saddle points with that of zeros,

$$
\begin{gathered}
x_{s} \simeq 3 \pi / 4-s \pi-(1 / 2) \arg \eta, \\
y_{s} \simeq(1 / 2) \log |\eta|, \\
\eta \equiv\left[1-4 \cos ^{2}(\nu \pi)\right] / 2 \cos (\nu \pi),
\end{gathered}
$$


and poles,

$$
\begin{gathered}
x_{s} \simeq \pi / 4-s \pi-(1 / 2) \arg \eta, \\
y_{s} \simeq(1 / 2) \log |\eta|,
\end{gathered}
$$

of $F_{\nu}(z)$ [2]. As can be seen, zeros and poles alternate along an almost horizontal line, with a separation of approximately $\pi / 2$ from each zero to neighboring poles. The line joining "upper" saddle points is above this zero-pole line and has a small negative slope, whereas that for "lower" saddle points is below and presents a small positive slope. As $\nu$ tends to a half-integer, saddle points go to infinity in the quadrant $-3 \pi / 2<\arg z<-\pi$, showing a behavior similar to that of poles (zeros) in the case of "upper" ("lower") saddle points. As $\nu$ increases towards $n+1 / 2$, the saddle points move almost vertically, tending to infinity along the asymptotes $x=(-s+n / 2+1 / 4) \pi$ in the case of "upper" ones and $x=(-s+n / 2-1 / 4) \pi$ in the case of "lower" ones, the label $s$ being larger than $n$. All saddle points at infinity jump by $\Delta x=\pi / 2$ as $\nu$ passes $n+1 / 2$ and go down along the asymptotes $x=(-s+n / 2+3 / 4) \pi$, if "upper", and $x=(-s+n / 2+1 / 4) \pi$ if "lower" saddle points.

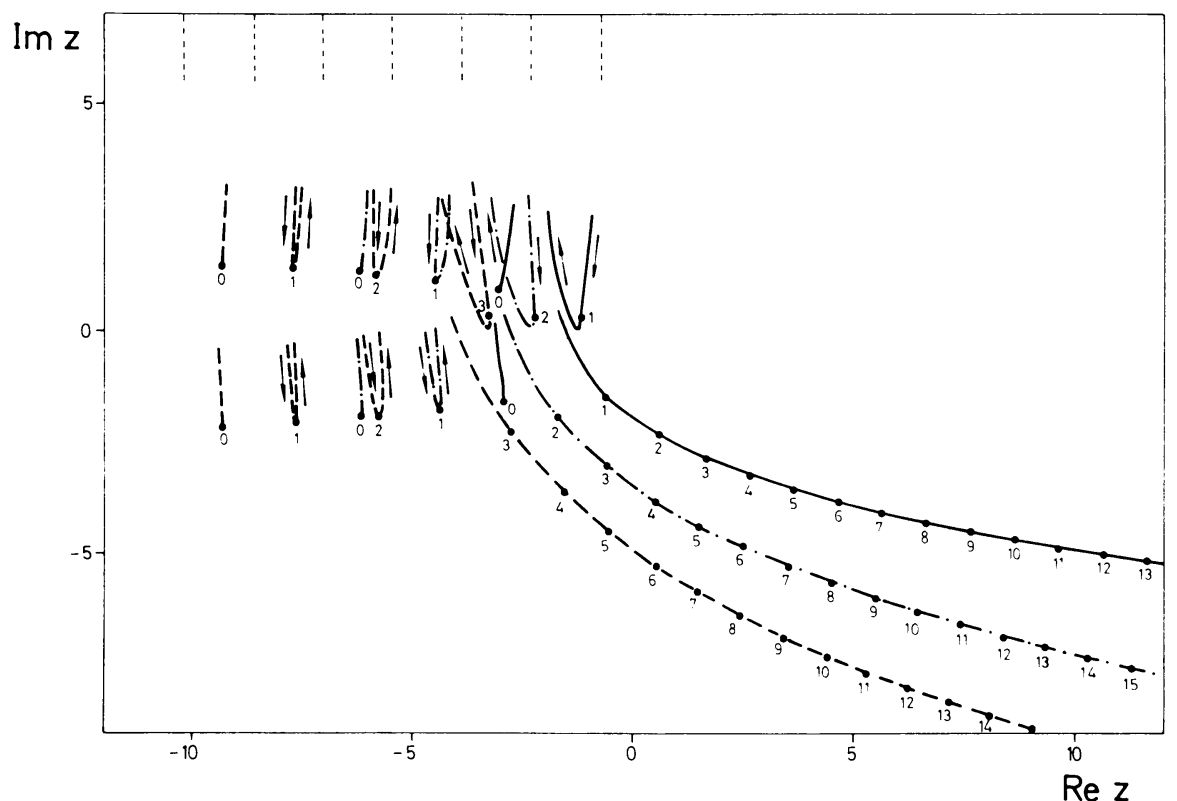

FIGURE 1

Saddle points of the reduced logarithmic derivative of the Hankel function. The trajectories followed by the first three pairs of saddle points, as the order $\nu$ varies from zero through positive values, are shown. The $z$ plane represented corresponds to the Riemann sheet $-3 \pi / 2<\arg z \leqslant \pi / 2$. The dots on the trajectories mark the positions of the saddle points at the integer values of $v$ indicated by numbers along the trajectories. The arrows correspond to the motion of saddle points along their trajectories with increasing $\nu$. The trajectories approach asymptotically the dashed straight lines in the top of the drawing.

It is possible to find analytic expressions giving the location of saddle points for values of $\nu$ not in the vicinity of a half-integer. A procedure due to Salzer [5] and used by Fettis et al. [3] to obtain the saddle points of the complementary error function was applied by the present authors [1] in the study of the saddle points of 
the reduced logarithmic derivative of the Bessel function. As Bessel and Hankel functions are solutions of the same differential equation, the analytic expressions giving the saddle points of the reduced logarithmic derivative of the Hankel function are formally the same as for the Bessel function, namely [1]

$$
z_{s}^{2}=z^{2}-b_{1} t-b_{2} t^{2}-b_{3} t^{3}-b_{4} t^{4} \ldots,
$$

where $t$ is a variable vanishing at the saddle points,

$$
t \equiv F_{\nu}(z)-\left(\nu^{2}-z^{2}\right)^{1 / 2},
$$

and having denoted

$(2.10, \mathrm{a}) \quad b_{1}=2 \mathrm{~g}$,

$(2.10, \mathrm{~b}) \quad b_{2}=1+2 g^{3} / z^{2}$,

$(2.10, \mathrm{c}) \quad b_{3}=\left(4 g^{2} / z^{2}\right)\left[1+\left(g^{2} / 3 z^{2}\right)(1+2 g)\right]$,

$(2.10, \mathrm{~d}) \quad b_{4}=\left(\mathrm{g} / 3 z^{2}\right)$

$$
\times\left\{15 / 2+\left(g^{2} / z^{2}\right)\left[10+31 g+\left(g^{2} / z^{2}\right)\left(4+14 g+12 g^{2}\right)\right]\right\},
$$

with

$$
g=\left(\nu^{2}-z^{2}\right)^{1 / 2}
$$

For a very precise determination of the position $z_{s}$ of a certain saddle point, one should start with an approximate value $z$ and apply the algorithm (2.8) repeatedly, so as to obtain $z_{s}$ with the desired accuracy.

3. Modulus and Phase Plots. We have represented in Figures 2 to 7 the contour lines in the $z$ plane corresponding to constant modulus or constant phase of $F_{\nu}(z)$ for $\nu=0,1 / 3,1 / 2,2 / 3,1$ and $3 / 2$, respectively. Those lines have been obtained numerically by expressing the Hankel function and its derivative in terms of Bessel functions, which were computed by means of their ascending series expansions. Double precision was used in the summation of the series. Of course, both families of modulus and phase lines are orthogonal.

In the far regions of the $z$ plane the constant-modulus lines resemble circumferences, their radial lines being the constant-phase ones. This was to be expected if one considered the asymptotic expansions [4, Eqs. 9.2.7 and 9.2.13] of the Hankel function and of its derivative. The corresponding approximate expression of the reduced logarithmic derivative becomes

$$
\begin{aligned}
F_{\nu}(z)=i z-1 / 2-i\left(4 v^{2}-1\right) & / 8 z+O\left(z^{-2}\right), \\
|z| & \rightarrow \infty,-\pi<\arg z<2 \pi,
\end{aligned}
$$

which shows that the (large) constant-modulus lines tend to be circumferences, with center at the point $-i / 2$, nearly independent of the value of $\nu$. Obviously, the approximate expression (3.1) is not valid in the region where poles and zeros lie about.

Our study of the behavior of zeros, poles and saddle points, presented in Section 2 , allows us to illustrate the evolution of the modulus and phase lines as $\nu$ varies. 


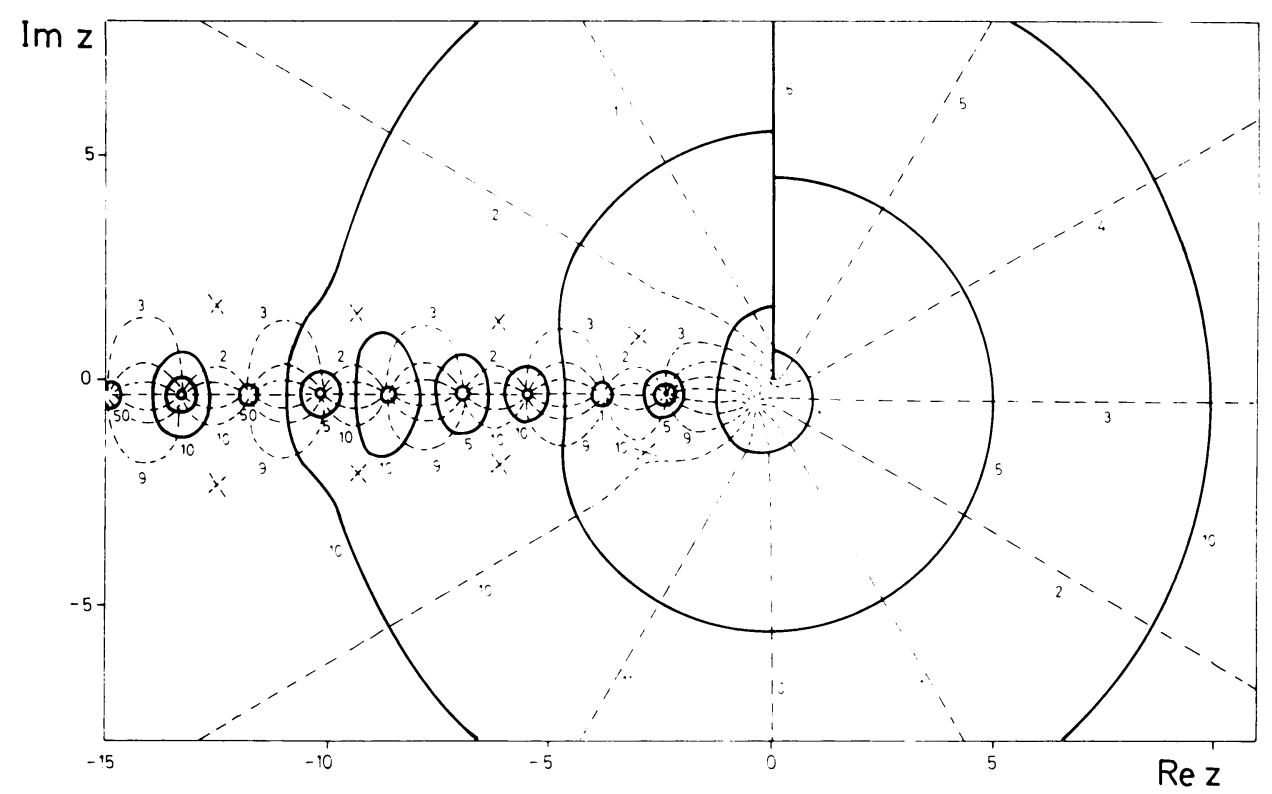

FIGURE 2

Modulus and phase of the reduced logarithmic derivative of the Hankel function of $\operatorname{order} \nu=0$. The $z$ plane shown corresponds to the Riemann sheet $-3 \pi / 2<\arg z \leqslant \pi / 2$. The cut along the positive imaginary semiaxis is represented by a solid line. The numbers aside the lines indicate the value of the modulus (continuous lines) or the phase in units $\pi / 6$ (dashed lines). Saddle points are indicated by the intersection of their constant-phase lines.

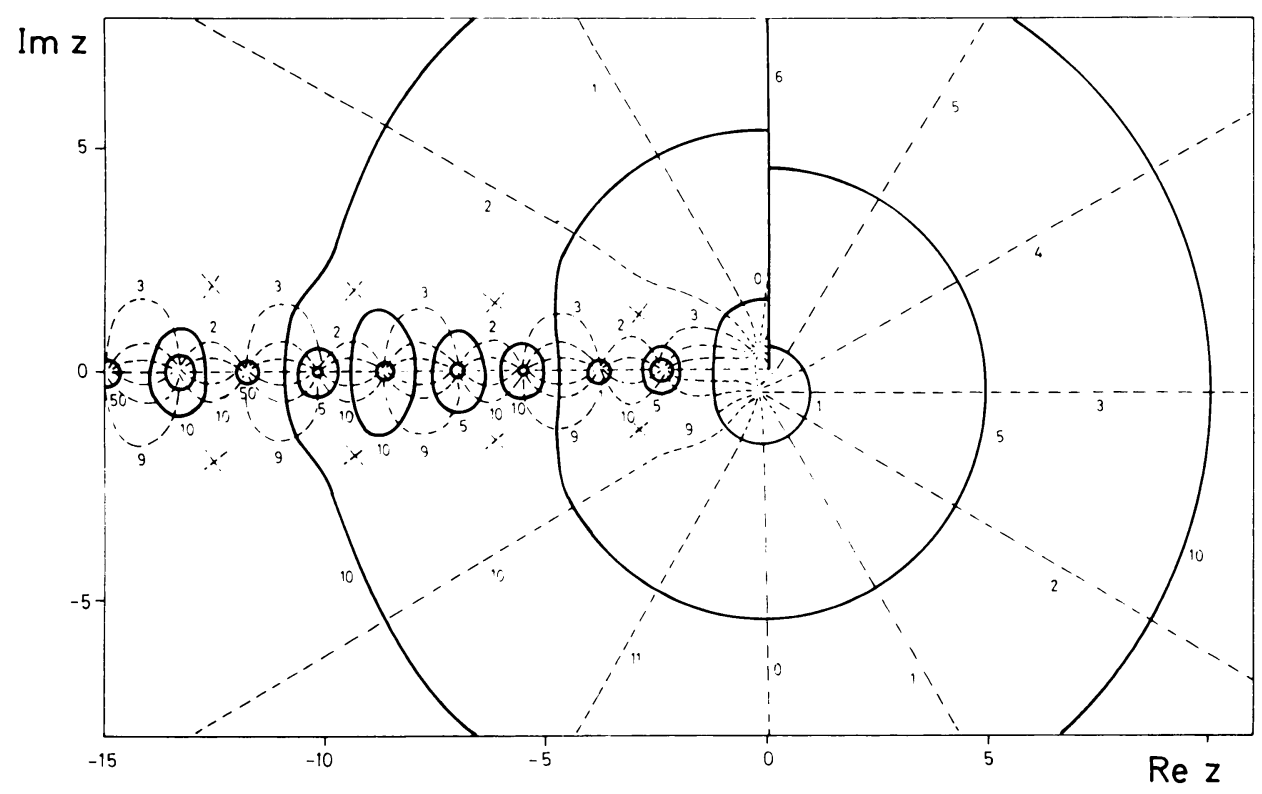

FIGURE 3

Modulus and phase of the reduced logarithmic derivative of the Hankel function of $\operatorname{order} \nu=1 / 3$. All the considerations made in the caption to Figure 2 are valid also here. Notice the symmetry with respect to the horizontal semiaxis in the left half-plane. 


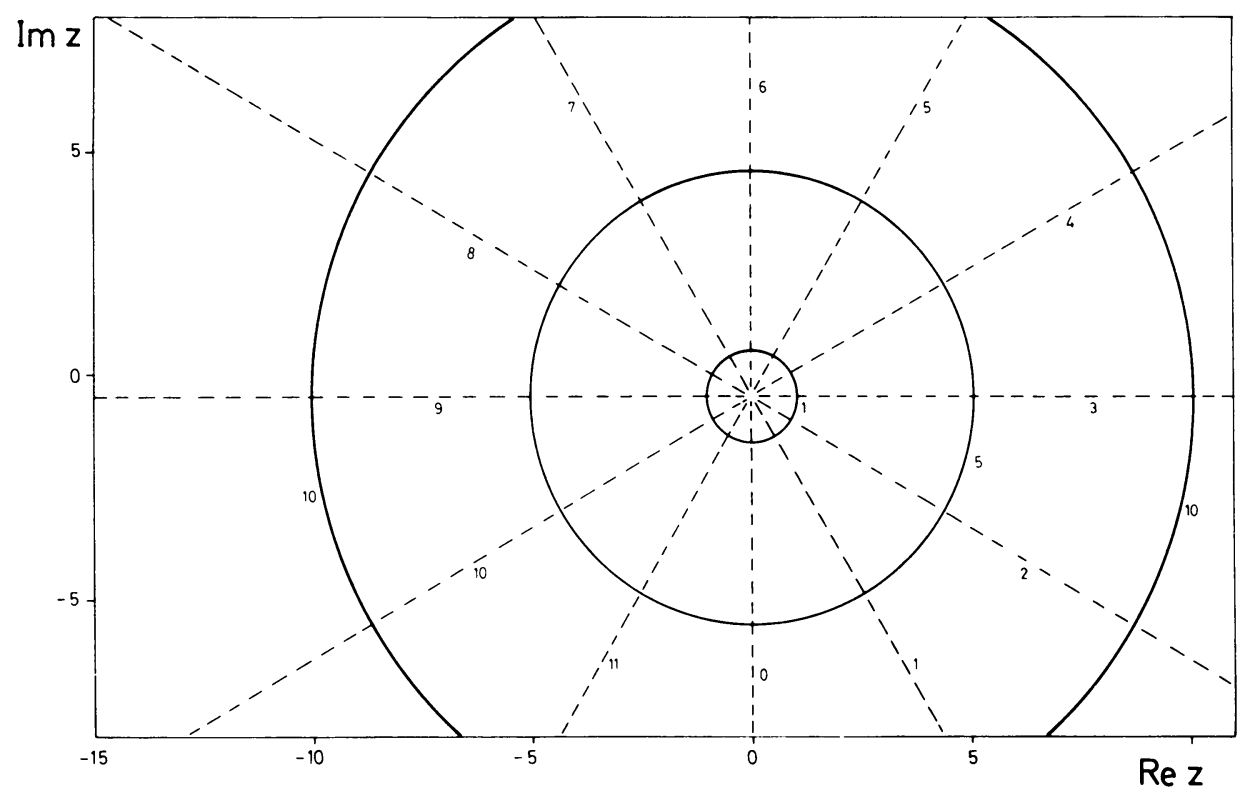

FIGURE 4

Modulus and phase of the reduced logarithmic derivative of the Hankel function of order $\nu=1 / 2$. The meaning of the symbols is the same as in Figure 2. As the represented function is singlevalued, no cut is needed. Since $F_{1 / 2}(z)=i z-1 / 2$, the constant-modulus lines are exactly circumferences with center at $-i / 2$, and the constant-phase ones radial straight lines.

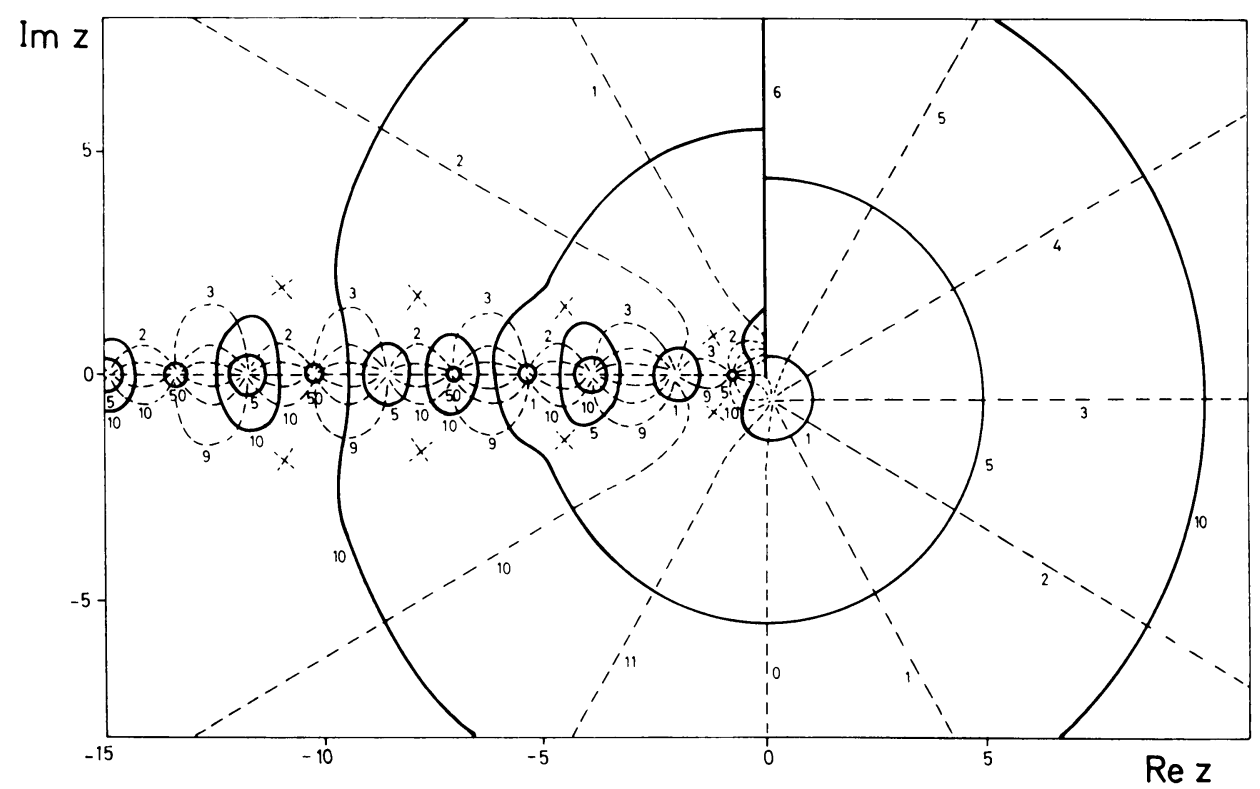

FIGURE 5

Modulus and phase of the reduced logarithmic derivative of the Hankel function of $\operatorname{order} \nu=2 / 3$. The comments to Figure 3 are applicable here. 


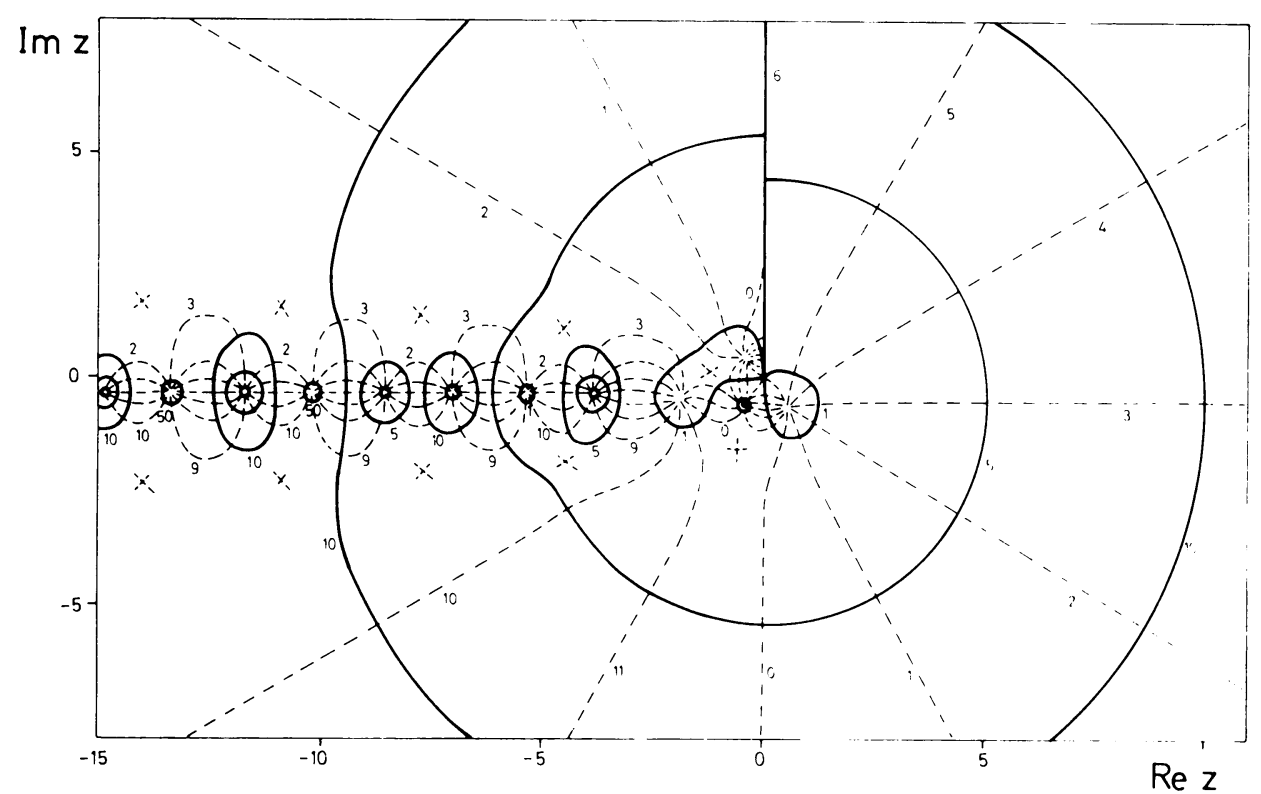

FIGURE 6

Modulus and phase of the reduced logarithmic derivative of the Hankel function of $\operatorname{order} \nu=1$. The same conventions as in Figure 2 have been adopted.

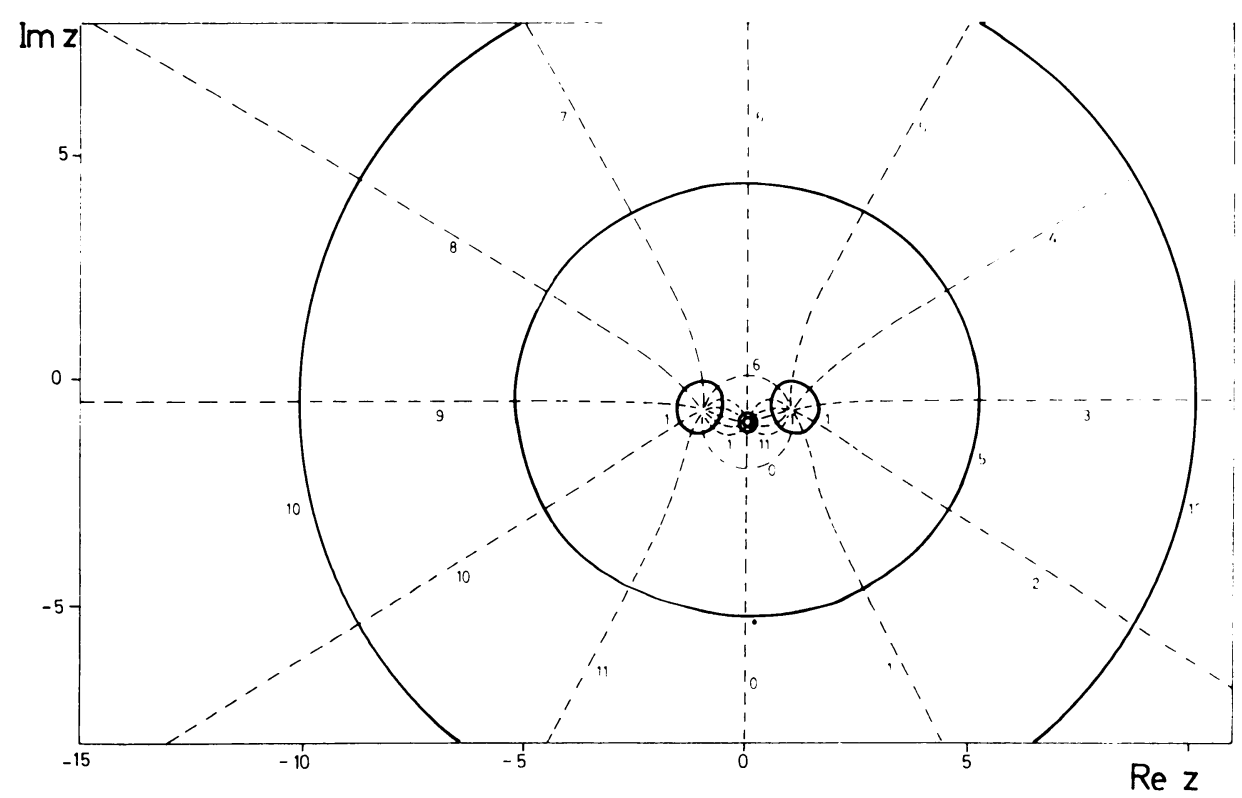

FIGURE 7

Modulus and phase of the reduced logarithmic derivative of the Hankel function of order $\nu=3 / 2$. As in Figure 4, the function is singlevalued and presents only a finite number of zeros and poles. 
Besides the circumference-like lines mentioned in the preceding paragraph, constant-modulus lines appear surrounding zeros and poles and constant-phase lines joining them. The set of zeros and poles and the mentioned lines shows the appearance of a horizontal "chain". For integer values, $n$, of $\nu$ the chain lies just below the negative real semiaxis (Figure 2). It moves upwards, remaining horizontal, as $\nu$ increases, crosses the real axis at $\nu=n+1 / 3$ (Figure 3) and goes to infinity, disappearing as $\nu$ reaches $n+1 / 2$ (Figure 4). As $\nu$ increases further, the chain, after suffering a displacement of $\pi / 2$ to the right, comes down from infinity, crosses the real axis at $\nu=n+2 / 3$ (Figure 5) and occupies the position corresponding to integer $\nu$ at $\nu=n+1$ (Figure 6).

The authors acknowledge the financial aid of Instituto de Estudios Nucleares.

Departamento de Física Teórica

Facultad de Ciencias

Zaragoza, Spain

1. A. Cruz \& J. Sesma, "Modulus and phase of the reduced logarithmic derivative of the cylindrical Bessel function," Math. Comp., v. 35, 1980, pp. 1317-1324.

2. A. Cruz \& J. Sesma, "Zeros of the Hankel function of real order and of its derivative," Math. Comp., v. 39, 1982, pp. 639-645.

3. H. E. Fettis, J. C. Caslin \& K. R. Cramer, "Saddle points of the complementary error function," Math. Comp., v. 27, 1973, pp. 409-412.

4. F. W. J. Olver, "Bessel functions of integer order," Handbook of Mathematical Functions (M. Abramowitz \& I. A. Stegun, Eds.), Dover, New York, 1965, pp. 355-433.

5. H. E. Salzer, "Complex zeros of the error function," J. Franklin Inst., v. 260, 1955, pp. 209-211. 